Recepción: 19 / 11 / 2018

Aceptación: 28 / 12 / 2018

(c) (i) (2)

Ciencias de la salud

Publicación: 20 / 02 / 2019

Artículo de Revision

\title{
Seguridad social y desempeño del personal hospitalario
}

\section{Social security and performance of hospital staff}

\section{Segurança social e desempenho do pessoal hospitalar}

Tatiana P. Vinces-Sornoza ${ }^{\mathrm{I}}$

tatianapaolavinces@gmail.com

Yandry M. Ponce-Menendez II

yanpo-005@hotmail.com

Beneda M. Zambrano-Mora ${ }^{\text {III }}$

ben_licen@hotmail.com

Diana L. Macias-Cedeño IV

maciasd395@gmail.com

José C. Pinargote-Menendez V

josec_pinoargote@hotmail.com

Cecilio A. Viteri-Uquillas VI

andrés.viteri@iica.int

Correspondencia: tatianapaolavinces@gmail.com

I. Licenciada en Enfermería; Universidad Tecnica de Manabi; Portoviejo, Ecuador.

II. Magister en Seguridad y Salud Ocupacional; Médico Cirujano; Hospital Verdi Cevallos Balda; Docente de la Universidad Tecnica de Manabi; Portoviejo, Ecuador.

III. Licenciada en Enfermería; Hospital de Especialidades, Portoviejo, Ecuador.

IV. Médico Cirujano; Profesional en la Práctica Privada; Portoviejo, Ecuador.

V. Médico; Ministerio de Salud Pública; Manta, Ecuador.

VI. Ingeniero Químico; Supervisor Planta en el Proyecto Piñón para Galápagos contrató por el IICA; Portoviejo, Ecuador. 


\title{
Resumen
}

Este trabajo tiene su basamento 13 revisiones bibliográficas consultadas de prestigiosas bases de datos como SCIELO y un gran alto nivel de actualización que abordan los aspectos de la Seguridad Social y Seguro Social, conceptos muchas veces no delimitados por algunos autores. Junto a esta reflexión se hace un bosquejo de las características de algunas Instituciones de Seguridad Social de América Latina, partiendo de los conceptos básicos de la Salud Ocupacional y se exponen algunos términos más usados que se encuentran registrados en las Leyes de Seguridad social de casi todos los países latinoamericanos; abordándose los riesgos laborales presentes específicamente en el entorno hospitalario y sus efectos en el personal de la Salud; finalmente se comenta su influencia en el desempeño profesional de estos trabajadores.

Palabras claves: Seguridad Social; Seguro Social; Salud Ocupacional; Prestaciones Sociales; Riesgos Hospitalarios; Desempeño Profesional.

\begin{abstract}
This work has its basement 13 bibliographic reviews consulted from prestigious databases such as SCIELO and a high level of updating that addresses aspects of Social Security and Social Security, concepts often not defined by some authors. Together with this reflection, an outline of the characteristics of some Social Security Institutions of Latin America is made, starting from the basic concepts of Occupational Health and some more used terms that are registered in the Social Security Laws of almost all are exposed the Latin American countries; addressing the occupational risks present specifically in the hospital environment and its effects on health personnel; Finally, its influence on the professional performance of these workers is discussed.
\end{abstract}

Keys words: Social Security; Social Security; Occupational Health; Social Benefits; Hospital Risks; Professional Performance.

\section{Resumo.}

Este trabalho tem como base 13 revisões bibliográficas consultadas em bancos de dados de prestígio como o SCIELO e um alto nível de atualização que aborda aspectos da Previdência Social e Previdência Social, conceitos muitas vezes não definidos por alguns autores. Juntamente com essa reflexão, faz-se um esboço das características de algumas Instituições de Previdência Social da América Latina, partindo dos conceitos básicos de Saúde Ocupacional e alguns termos mais 
Tatiana P. Vinces-Sornoza; Yandry M. Ponce-Menendez; Beneda M. Zambrano-Mora; Diana L. Macias-Cedeño; José C. Pinargote-Menendez; Cecilio A. Viteri-Uquillas

utilizados que estão registrados nas Leis de Previdência Social de quase todos estão expostos a América Latina. países; abordar os riscos ocupacionais presentes especificamente no ambiente hospitalar e seus efeitos no pessoal de saúde; Por fim, discute-se sua influência no desempenho profissional desses trabalhadores.

Palavras chaves: Segurança social; Seguro Social; Saúde Ocupacional; Prestações sociais; Riscos Hospitalares; Desempenho profissional.

\section{Introducción.}

La actividad humana además de ayudar a transformar al hombre de las cavernas en el técnico, profesional o científico de hoy contribuyó a desarrollar su cerebro, pero también representa en ocasiones un riesgo importante de sufrir accidentes o enfermedades ocasionadas por los materiales, herramientas y equipos empleados en el trabajo o por los productos o subproductos que se elaboran.

Larga es la historia de la Salud Ocupacional, desde antes de nuestra era con las observaciones de Hipócrates, hasta nuestros días; pasando por el médico italiano Bernardino Ramazzini (1633-1714) que con su obra magistral "De morbis artificium diatriba (De las enfermedades de los trabajadores) describió magistralmente las enfermedades que afectaban a los trabajadores de los numerosos oficios conocidos en aquella época, haciendo observaciones precisas y aún vigentes en cierta forma.

Con la Revolución industrial en el siglo XVIII, aparece una nueva y valiosa herramienta tecnológica y económica produciéndose una verdadera revolución económica, social y moral; esta organización de la nueva industria representó una verdadera tragedia para las clases laborales ya que las condiciones de trabajo no eran las mejores; los salarios alcanzaban a penas para adquirir alimentos y vestuario para el obrero y su familia; los accidentes de trabajo y las enfermedades ocupacionales diezmaban a los obreros; por lo que constituyó una motivación para los trabajadores comenzar los movimientos sociales en los primeros años del siglo XX. Los intentos de la lucha de los obreros 
consistieron en la protección de los mismos, organizándose los Servicios de Salud Ocupacional, siendo los primeros en América Latina los de Perú, Bolivia, Colombia, Venezuela y otros.

La misión fundamental de esta disciplina es la protección de la salud de los trabajadores contra los peligros producidos por el ambiente en que desempeñan sus funciones. Este es el punto de vista de mayor interés: el humano y el social.

\section{Metodología.}

Se realizó una revisión bibliográfica en la cual se consultaron 9 referencias bibliográficas de prestigiosas revistas que tienen un alto grado de actualización con respecto al tema.

\section{Desarrollo.}

\section{Definición e importancia de la Salud Ocupacional}

Diversas son las definiciones de Salud Ocupacional, pero el Comité de expertos en Salud Ocupacional de la Organización Internacional del Trabajo (OIT) y la Organización Mundial de la Salud (OMS) la definieron en 1950, de a siguiente manera; Cito (...) “ tiene como finalidad promover y mantener el más alto grado de bienestar fîsico, mental y social de los trabajadores en todas las profesiones, evitar el desmejoramiento de la salud causado por las condiciones de trabajo; protegerlos en sus ocupaciones de los riesgos resultantes de los agentes nocivos; ubicar y mantener a los trabajadores de manera adecuada a sus aptitudes fisiológicas y psicológicas y, en suma, adaptar el trabajo al hombre y cada hombre a su trabajo" 
Tatiana P. Vinces-Sornoza; Yandry M. Ponce-Menendez; Beneda M. Zambrano-Mora; Diana L. Macias-Cedeño; José C. Pinargote-Menendez; Cecilio A. Viteri-Uquillas

Esta definición nos lleva a plantear la importancia de la disciplina; las consecuencias para la salud, que son causas de incapacidades que sufren los trabajadores y las consecuencias económicas. Las primeras están dadas por:

- Accidentes de trabajo.

- Accidentes ocurridos fuera del ambiente laboral.

- Enfermedades ocupacionales u profesionales.

- Enfermedades comunes.

Es importante destacar la carga económica que representan los accidentes y enfermedades en la fuerza laboral, dada la influencia cada vez mayor del desarrollo económico para los países latinoamericanos y para el bienestar de los ciudadanos; por tanto, las consecuencias para la economía están dadas por:

- El valor de los equipos y las maquinarias destruidos o deteriorados.

- Las materias primas y los productos elaborados dañados.

- El costo de la atención medica.

- De las compensaciones pagadas.

- Lo que dejan de ganar los trabajadores afectados.

- Otros.

Son estas y mucho más las razones que conllevaron a crearse los Seguros Sociales, así como las instituciones encargadas del análisis de las consecuencias económicas. 
Concepto de Seguridad Social

La Seguridad Social, también llamada Previsión Social, describe principalmente a un campo de bienestar social relacionado con la protección social o cobertura de las necesidades reconocidas socialmente, como la salud, la vejez o las discapacidades La Organización Internacional del Trabajo (OIT), en el documento denominado "Administración de la seguridad social", definió la seguridad social como; Cito (...) “Es la protección que la sociedad proporciona a sus miembros, mediante una serie de medidas públicas, contra las privaciones económicas y sociales que, de no ser así, ocasionarían la desaparición o una fuerte reducción de los ingresos por causa de enfermedad, maternidad, accidente de trabajo, o enfermedad laboral, desempleo, invalidez, vejez y muerte; también la protección en forma de asistencia médica y de ayuda a las familias con hijos" 1

\section{Concepto de Seguro Social}

El seguro social es un sistema de protección contra las contingencias que da cobertura a la población que mantiene una relación laboral y se encuentra financiada por los aportes de trabajadores, empleadores y Estado. Es un mecanismo por el cual se ejecuta la seguridad social.

La Enciclopedia ILO (International Labour Office), expone que: Cito (...) la expresión "seguros sociales" se refiere usualmente a un régimen de seguros, administrado por el gobierno, con cobertura obligatoria y cotizaciones que han de pagar las empresas, los trabajadores o ambos, aun cuando puede existir una aportación de los ingresos públicos generales. Este régimen normalmente cubre a los trabajadores asalariados, aunque pueden acogerse los trabajadores por cuenta propia; mientras que la expresión "seguridad social" tiene varios significados: por un lado, suele referirse a un régimen de prestaciones pagadas por el Gobierno con los ingresos públicos generales a personas que pueden optar a ellas por motivo de incapacidad, vejez, desempleo y por otro lado incluye a estas 
Tatiana P. Vinces-Sornoza; Yandry M. Ponce-Menendez; Beneda M. Zambrano-Mora; Diana L. Macias-Cedeño; José C. Pinargote-Menendez; Cecilio A. Viteri-Uquillas

prestaciones más los seguros sociales, la asistencia médica y los servicios sociales. Estos aspectos se desarrollan y pueden ser consultados con más detalles en tratados sobre seguridad social y seguros sociales (Organización Internacional del Trabajo (OIT)).

La Organización Internacional del Trabajo junto a la Organización Mundial dela Salud (OMS) han construido el concepto de Piso de Protección Social entendido como; Cito (...), "un conjunto integrado de políticas sociales diseñado para garantizar a todas las personas la seguridad de los Ingresos y el acceso a los servicios sociales esenciales, prestando especial atención a los grupos más desprotegidos, capacitando a las personas a lo largo del ciclo de vida"; (Organización Internacional del Trabajo (OIT))

\section{Instituciones de Seguridad Social}

Los modelos de seguridad social ensayados para América Latina no fueron pensados desde su misma realidad, en todos ellos se puede rastrear su origen ya sea en el modelo alemán de Bismarck, basado en los seguros sociales dirigido a trabajadores fabriles, o en el modelo inglés ideado por Beveridge, cimentadoen la construcción de un sistema nacional de salud universal y prestaciones mínimas para todos sin importar su condición laboral.

En toda Latinoamérica existen los Institutos de Seguridad Social con las características propias de cada país, así tenemos en México, el Instituto Mexicano de Seguro social (IMSS); en éste país el Estado proporciona la seguridad social a través del servicio público que se encarga de suministrar el esquema de protección e la forma y términos que establece la ley de Seguridad social; este servicio es nacional, definido, contributivo, con soporte actuarial, eminentemente obligatorio y con administración tripartita. Esta ley menciona 2 rubros: régimen obligatorio y régimen voluntario. (Moreno, 2016) 
En Chile existe el Instituto de Seguridad Laboral que otorga prestaciones económicas en el caso de un accidente de trabajo, de trayecto o enfermedad profesional, que ocasione a un trabajador(a) adherido al éste algún grado de incapacidad, igual o superior a un $15 \%$, o la muerte ${ }^{3}$. (Instituto de Seguridad Laboral, 2016)

En Ecuador es adoptado el modelo de seguros sociales, desde 1928 con la creación de la Caja de Pensiones y luego de varios años de variadas modificaciones se llega hasta la Ley de la Seguridad Social publicada en el Registro oficial No.465 en el 2001, vigente hasta la actualidad y la Constitución de 2008 que incluye algunas novedades en cuanto a la seguridad social. En el año 2001 se crea el Seguro General Obligatorio y dentro de este los regímenes de Seguro Voluntario y el Seguro Social Campesino; ambos son de carácter contributivo, es decir, son sistemas cuyo financiamiento depende de los aportes que entreguen los trabajadores, empleadores y ciertas contribuciones del Estado. El primero está sobre todo encaminado a la cobertura de quienes desarrollan alguna actividad económica ya sea como trabajadores dependientes o autónomos; y el segundo, estuvo diseñado para todos aquellos que no se incluyan en el primero. En cuanto al seguro social campesino es un régimen semicontributivo que se sustenta con una pequeña contribución del jefe o jefa de familia y se complementa con el aporte de los trabajadores afiliados y otros ingresos.

La atención de la salud está garantizada por el Instituto Ecuatoriano de Seguridad Social (IESS) que le brinda prestaciones en las unidades de salud a los jubilados también; son varias estas unidades por todo el país, como se observa en la siguiente figura. 


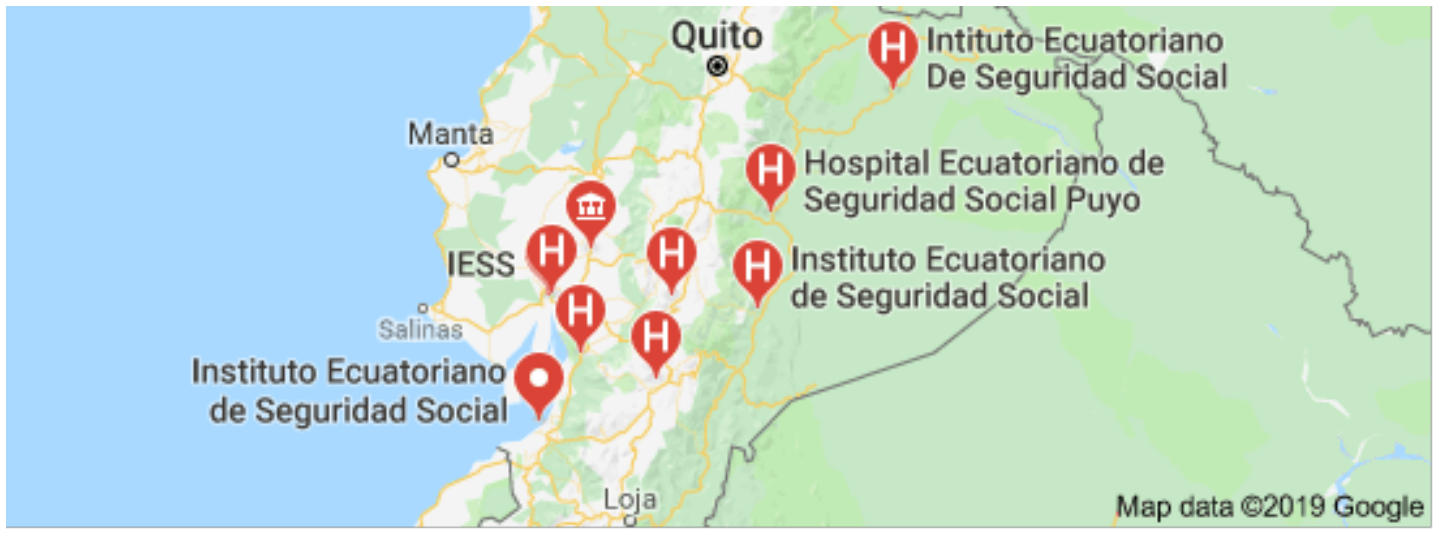

Fuente: Hospitales del IESS (Porras, 2015)

En Colombia el acceso a los servicios de salud incluido el de urgencia se garantiza por medio del Sistema General de Seguridad Social en Salud (SGSSS) el cual cubre la población del país mediante tres regímenes, a saber: Contributivo, Subsidiado y Especiales; en lo referente al contributivo, se afilian los trabajadores formalmente empleados, los pensionados y los trabajadores independientes, quienes se denominan cotizantes y los cuales tienen derecho a incluir a los miembros de su grupo familiar, a quienes se les denomina beneficiarios. Al régimen Subsidiado se afilian los grupos familiares de personas de bajo estrato socioeconómico usualmente en el mercado laboral informal con una reducida o nula capacidad de pago. (Rodríguez-Páez, Jiménez-Barbosa, \& PalenciaSánchez, 2018)

En Venezuela la seguridad social es un derecho humano y social fundamental e irrenunciable, garantizado por el Estado a todos los venezolanos residentes en el territorio de la República, y a los extranjeros residenciados legalmente en él independientemente de su capacidad contributiva, condición social, actividad laboral, medio de desenvolvimiento, salarios, ingresos y renta, conforme al principio de progresividad y a los términos establecidos en la Constitución y las leyes venezolanas. La seguridad social integra en todo su ámbito al seguro social a través del Instituto 
Venezolano de los Seguros Sociales (IVSS), la asistencia social, las prestaciones que se financian con los ingresos generales del Estado, las asignaciones familiares y su protección (Coromoto, 2015)

El Instituto Nicaragüense de Seguridad Social (INSS), concibe que toda persona trabajadora inscrita a él o que cotiza al Seguro Social en la empresa donde trabaja, cuenta con el Seguro de Riesgo Profesional, entendiendo por éste a los accidentes y las enfermedades a que están expuestas los trabajador@s en ocasión del trabajo; que los protege ante las contingencias derivadas de su actividad laboral y repara el daño económico que pudieran causarle a ella o a sus familiares, amparándose en el capítulo IV artículo 60 de la Ley de Seguridad Social, garantizando las prestaciones en salud y económicas, tanto por accidente de trabajo o en el trayecto hacia el mismo, como por enfermedad laboral.

El Seguro de Riesgo Profesional cubre prestaciones de salud como: - atención Médica y quirúrgica y diferenciada desde la emergencia, hospitalización y consulta externa, hasta su total recuperación; servicio de apoyo diagnóstico y terapéutico que requiere el asegurado; - medicamentos sin exclusión hasta la resolución del caso. - atención en Rehabilitación Física y Psicosocial; - Ortèsis y Prótesis (silla de rueda, muletas, cabestrillo, etc.); atención Odontológica, - atención Odontológica y oftalmológica a consecuencia de accidente de trabajo o trayecto y enfermedad profesional.

El Seguro de Riesgo Profesional también cubre prestaciones económicas como el subsidio por incapacidad temporal para el trabajo, indemnizaciones, pensiones por incapacidad permanente, parcial o total; pensiones por muerte: viudez, orfandad, ascendientes u otros, además de subsidio funeral (Insitituto Nicaraguense de Seguro Social , 2018)

Algunos términos utilizados en la Ley de Seguridad Social 
Tatiana P. Vinces-Sornoza; Yandry M. Ponce-Menendez; Beneda M. Zambrano-Mora; Diana L. Macias-Cedeño; José C. Pinargote-Menendez; Cecilio A. Viteri-Uquillas

Pensión: Es la prestación en dinero, en forma de renta temporal o vitalicia que el Seguro Social paga mensualmente a sus asegurados o a los beneficiarios de éstos, previo cumplimiento de los requisitos legales correspondientes. La Caja de Seguro Social otorga las pensiones a través de tres programas a saber:

a. Programa de Invalidez, Vejez y Muerte: formado por las pensiones de Vejez, Vejez Anticipada, Invalidez y Sobreviviente.

b. Programa de Riesgos Profesionales: formado por las Pensiones Parcial Permanente, Absoluta Permanente y la Pensión de Sobreviviente.

En América latina a mediados de los años 90 del siglo pasado aparece la llamada pensiones no contributivas, como su nombre lo indica, son aquellas que no requieren la cotización previa a la seguridad social, consisten en "prestaciones monetarias relativamente uniformes en forma focalizada y/o categórica ante los riesgos de vejez, discapacidad e invalidez". Las características más distintivas de este modelo, se refieren a que las condiciones de acceso están generalmente desvinculadas de la trayectoria laboral y el financiamiento proviene de impuestos generales.

- Pensionado: Es la persona que recibe una pensión del Seguro Social adquirida, en virtud de sus propios aportes en calidad de trabajador cotizante; esta comprende los pensionados por riesgos de trabajo, invalidez y vejez.

- Prestación: Es cualquier tipo de beneficio que, de acuerdo a su ley y reglamento, otorga el Seguro Social a los asegurados cotizantes o a los beneficiarios. Se clasifican en prestaciones médicas, en dinero y en especies. 
- Prestación por enfermedad: Se concede cuando el asegurado se encuentra en un estado que le impide desempeñar sus funciones profesionales debido a la alteración de su salud física o mental.

\section{La Caja concederá las siguientes prestaciones:}

a) Por maternidad: Las aseguradas tendrán derecho en el curso del embarazo, el parto y el puerperio, a la asistencia prenatal y obstétrica que requiera su estado, independientemente de las prestaciones asistenciales a que puedan tener derecho en caso de enfermedad.

b) Subsidio: Es la prestación en dinero que recibe en forma periódica y temporal el asegurado con derecho, por concepto de enfermedad común, accidente común, de trabajo, maternidad o enfermedad profesional. Cubre los días de incapacidad para el trabajo, de conformidad con la certificación médica extendida por un facultativo al servicio del Seguro Social.

Los subsidios concedidos por la Caja de Seguro Social se otorgan, a través de tres programas:

a. Enfermedad y Maternidad formado por los subsidios de Incapacidad Temporal, Maternidad y Lentes.

b. Invalidez, Vejez y Muerte que concede los subsidios de Funeral y Escolaridad.

c. Riesgos Profesionales, que otorga los subsidios de Incapacidad Temporal y Funeral.

- Accidente de trabajo: Es toda lesión corporal o perturbación funcional que el trabajador sufra, sea en la ejecución, con ocasión o por consecuencia del trabajo, y que dicha perturbación sea producida por la acción repentina o violenta de una causa exterior, o del esfuerzo realizado. 
Tatiana P. Vinces-Sornoza; Yandry M. Ponce-Menendez; Beneda M. Zambrano-Mora; Diana L. Macias-Cedeño; José C. Pinargote-Menendez; Cecilio A. Viteri-Uquillas

- Enfermedad Profesional: Todo estado patológico que se manifieste de manera súbita o por evolución lenta, a consecuencia del proceso de trabajo o debido a las condiciones específicas en que éste se ejecute.

Estos elementos conceptuales de la Seguridad Social son aplicables a cualquier institución o centro laboral, tanto de producción como de servicios, en el caso de los servicios hospitalarios, es bueno recordar los diversos riesgos laborales que se encuentran presentes en estas unidades de salud, y que son capaces de producir accidentes laborales o enfermedades profesionales sin descartar las enfermedades comunes; todas con una gran carga de ausentismo laboral y costo económico.

\section{Riesgos laborales hospitalarios}

Existen ciertas características en las instituciones de salud que justifican un estudio particular, que abarca tanto a los públicos como a los privados; asi como aquellos centros de la Atención Primaria y centros de diagnóstico. Nos ocuparemos en este artículo de la atención secundaria y terciaria, pues las consecuencias de los riesgos influyen en el Sistema de Seguridad Social por los daños que ocasionan los accidentes y enfermedades, ya sean comunes o profesionales a los trabajadores.

El término "personal hospitalario" que aparece en diversas publicaciones sobre el tema, agrupa categorías muy diferentes: administrativas, encargados de la gerencia o dirección, los cuales no presentan particularidades en la prevención; obreros expuestos a los riesgos inherentes a sus empleos; personal hospitalario encargado de manera específica del cuidado de los enfermos; nos referimos a los médicos, enfermeras y técnicos de medios diagnósticos.

Los médicos, enfermeras, especialistas y auxiliares hospitalarios tienen en común los riesgos de infección y agresión por agentes biológicos y químicos y el estrés psíquico debido al trabajo; 
téngase en cuenta que las dos terceras partes del personal es femenino las que comportan situaciones particularidades en el plano psicológico y social.

Veremos los accidentes de trabajo; estos aparecen con mayor frecuencia en los servicios técnicos, tales como cortes en los dedos, quemaduras en los cocineros; excoriaciones y cuerpos extraños metálicos en los ojos y en partes blandas en los mecánicos; sacrolumbalgias en los camilleros y transportistas; caídas y accidentes de circulación en todos ellos, etc; pero un personal totalmente expuestos a estos riesgos es el personal de enfermería y de laboratorio; aunque estudio realizado en un hospital público de Argentina ha resultado que los médicos portan la mayor frecuencia, no obstante, es reconocido que los grupos de trabajadores mayormente afectados son los médicos y enfermeros, evidenciándose diferencias según las áreas o divisiones hospitalarias, tales como quirúrgicas, de emergencias, consultorios externos, laboratorios.

Datos obtenidos en sistema computadorizados resaltan que, en un hospital promedio, los trabajadores sufren aproximadamente 30 lesiones por pinchazos por cada 100 camas al año. Las lesiones por pinchazos reportadas con más frecuencia afectan al personal de enfermería, laboratorio, médicos, personal de mantenimiento fundamentalmente; algunas de estas lesiones exponen a los trabajadores a patógenos que pueden transmitir infecciones por Virus de la hepatitis B (VHB), Virus de la hepatitis C (VHC) y el Virus de la Inmunodeficiencia humana (VIH) fundamentalmente. El riesgo de infección después de una sola cortada con un objeto punzocortante contaminado es: VIH: 0.3\%, VHC: 1.8\% y VHB: 6-30\%. (Porras, 2015) Estos accidentes generalmente no causan pérdida de días por lo que frecuentemente no son registrados ni analizadas sus causas. Las lesiones por pinchazos profundos siguen siendo una causa importante y constante de exposición a enfermedades graves (en ocasiones mortales) en los trabajadores sanitarios. 
Tatiana P. Vinces-Sornoza; Yandry M. Ponce-Menendez; Beneda M. Zambrano-Mora; Diana L. Macias-Cedeño; José C. Pinargote-Menendez; Cecilio A. Viteri-Uquillas

Estudio realizado en instituciones de salud de la provincia de Pinar del Rio, Cuba arrojó que los trabajadores investigados tenían un elevado riesgo de accidentalidad mientras laboran manipulando sangre y sus derivados al representar este tipo de fluido el $90,8 \%$ del total de los trabajadores estudiados. (Padrón, Moreno, Márquez, González , \& Pérez, 2017) (Huanca, y otros, 2016)

En cuanto a las enfermedades profesionales, todas aquellas cuyas causas están dadas por un agente biológico, como las hepatitis víricas, enfermedades parasitarias, las dermatosis, alergias respiratorias, intoxicaciones, etc. un elemento a tener en cuenta es la higiene hospitalaria donde el uso de agentes químicos y físicos en los procesos de limpieza y desinfección debe hacerse siempre siguiendo las indicaciones del fabricante y las recomendaciones y protocolos establecidos en cada centro, ya que de lo contrario pueden generar riesgos para la salud de los profesionales. La Ley de Prevención de Riesgos Laborales subraya la importancia de adoptar medidas preventivas en el entorno laboral.

Dentro de los impactos sociales de la aplicación adecuada de esas normas o protocolo esta la disminución de bajas laborales debidas a las infecciones relacionadas con la asistencia sanitaria (IRAS); la mejora en la calidad y la seguridad del paciente, y la consiguiente reducción de la probabilidad de adquirir una infección en el entorno hospitalario producirán una reducción en las bajas laborales ocasionadas hasta el momento, tanto en pacientes como en profesionales del centro sanitario y ésta disminución de las bajas laborales conlleva a su vez, a un aumento de la productividad laboral y un menor gasto social en prestaciones por incapacidad temporal.

La incapacidad temporal (IT) es aquella situación en la que el trabajador se encuentra ausente de su puesto de trabajo por motivos de salud, ya sea una enfermedad o lesión por accidente relacionada o no con el trabajo que lo imposibilita a trabajar de forma temporal y precisa de asistencia sanitaria; 
reducir el número de días que un trabajador se encuentra en situación de IT es un objetivo que comparten trabajadores, empresas, mutuas laborales y los sistemas de salud y protección social, tanto por la pérdida de salud y productividad, como por los costes asociados, y la menor probabilidad de retorno al trabajo a medida que avanza el episodio. Estudio realizado por Vilar Dell y colb, sobre la IT en 30 centros sanitarios de Cataluña, con un total 25.964 trabajadores investigados, muestran que las tasas de ITs de causa común, fueron muy superiores a los de causas profesionales, donde la mayoría fueron por accidentes laborales y una pequeña proporción por enfermedades profesionales. (Vilar, y otros, 2016)

¿Cómo influyen estos perfiles de la Seguridad Social con el desempeño profesional del personal de salud en un hospital?

El desempeño define el rendimiento laboral, es decir, la capacidad de una persona para producir, hacer, elaborar, acabar y generar trabajo en menos tiempo, con menor esfuerzo y mejor calidad, estando dirigido a la evaluación la cual dará como resultado su desenvolvimiento. Es el comportamiento o conducta real del trabajador para desarrollar competentemente sus deberes $\mathrm{u}$ obligaciones inherentes a un puesto de trabajo, es lo que hace no lo que sabe hacer, comprende la peripecia técnica, la preparación técnica más la experiencia acumulada, eficacia en decisiones, destreza en la ejecución de procedimientos y buenas relaciones interpersonales, entre otros. En resumen, son en realidad las acciones y funciones que realiza en su área de trabajo, esto con base a sus experiencias y conocimientos que obtiene durante su trayectoria académica o laboral, es importante dar a conocer que incluye las acciones intangibles como su creatividad en el trabajo.

Entre los factores que influyen en el desempeño laboral están: 
Tatiana P. Vinces-Sornoza; Yandry M. Ponce-Menendez; Beneda M. Zambrano-Mora; Diana L. Macias-Cedeño; José C. Pinargote-Menendez; Cecilio A. Viteri-Uquillas

- Las recompensas por el rendimiento. Se refiere al sistema de salarios y políticas de ascensos que se tiene en la organización. Se dice que el incentivo más poderoso que pueden utilizar los jefes o supervisores es el reconocimiento personalizado e inmediato.

- El dinero (salario). Es un incentivo complejo, uno de los motivos importantes por los cuales trabaja la mayoría de las personas, que tiene significado distinto para ellos. Se le considera como un reforzador universal y la gente trabaja porque el dinero es un medio para obtener cosas.

- La necesidad de capacitación, sinónimo de entrenamiento. Surge de los rápidos cambios ambientales, el mejorar la calidad de los productos y servicios e incrementar la productividad.

- La participación, colaboración y la interacción social (relaciones interpersonales). Los hombres trabajan porque se sienten bien con sus compañeros de trabajo, con los supervisores y los jefes.

- Condiciones físicas, ambientales, materias primas, las instalaciones y el ambiente general de una organización. El lugar de trabajo debe ser un sitio cómodo, acogedor, donde las personas deseen pasar tiempo en vez de huir.

En general, si no se siente satisfecho con el trabajo que realiza a diario o su entorno no podrá dar más en su desempeño profesional.

Estudio realizado en base al contexto económico-social del personal de Enfermería en Colombia se observa un deterioro de las condiciones de trabajo y de vida del personal de enfermería, en razón a que se pasó de los sistemas de contratación fijos a los flexibles y provisionales, lo cual ha 
representado pérdida de la estabilidad laboral, incertidumbre, precariedad y desatención de la seguridad e higiene del trabajo y la seguridad social.

En otra investigación con enfermeras en Chile en el año 2014, arrojó que es cada vez más común que se presenten contrataciones eventuales en instituciones públicas y/o privadas, lo que repercute en la estabilidad laboral y obstaculiza a este personal a reclamar sus derechos, debido a que en ocasiones el hecho de ser contratad@s de forma eventual o indeterminada, imposibilita el acceso a prestaciones laborales básicas, como ingresar a sindicatos, períodos vacacionales, incapacitarse en caso de enfermedad, etc.

De esta forma, la estabilidad laboral en enfermería u otro `personal de la salud, es factor clave para la calidad del cuidado y calidad de atención que brinda a diario, también el estado emocional positivo o negativo según sea su experiencia laboral, incide fuertemente en el desempeño en sus diferentes actividades de trabajo. Si se tiene estabilidad laboral es del conocimiento que el desempeño laboral será mejor (Martin, 2015)

Una de las patologías frecuentes en el ambiente hospitalario son los disturbios osteomusculares relacionados al trabajo (DORT) que causan disfunción e incapacidad e imponen altos costos con auxilio por enfermedad, siendo reconocidos como uno de los mayores responsables del alto absentismo, causas de bajas y la reducción de productividad; otros investigadores como Dias da Silvay colb. encontraron en su investigación que el 37,33\% de los profesionales que sintieron incomodidad en los brazos o cuello presentaron pocos períodso de baja, con la consiguiente aplicación de las prestaciones del seguro social. Esa alta prevalencia de incomodidad y el bajo número de bajas, pero con gran cantidad de éstas mayores que 15 días, es explicado porque los enfermer@s juegan un rol importante en la supervisión del equipo de enfermería y por tanto tardan 
Tatiana P. Vinces-Sornoza; Yandry M. Ponce-Menendez; Beneda M. Zambrano-Mora; Diana L. Macias-Cedeño; José C. Pinargote-Menendez; Cecilio A. Viteri-Uquillas

más en buscar atención médica; otro motivo fue que los enfermeras optan por trabajar, aun comprometidos por enfermedades (presentismo), a fin de no causar perjuicio al equipo. (Silva, Araújo, Stival, Toledo, Burke, \& Carregaro, 2018)

Otros factores del ambiente laboral lo constituyen los psicológicos, como es la fatiga inducida por el trabajo que ha sido reconocida por su importante papel en la etiología de sobrecargas psicofísicas. Recientemente, estudios realizados han detectados fatiga en el marco de la enfermería' los que han demostrado una alta prevalencia y asociación de esa variable con condiciones deletéreas de trabajo; la fatiga representa un proceso de agotamiento físico y psíquico que modifica el estado de alerta y vigilancia, afectando las habilidades y comprometiendo el desarrollo de las actividades ocupacionales. Esta profesión tolera una gran responsabilidad, enfrentándose en forma cotidiana a presiones, conflictos y cambios continuos que conllevarían tras varios años de actividad laboral, a ser afectados por el Síndrome de Burnout, causando ausentismo e incapacidad. Es extensa la lista de investigaciones de este síndrome en el personal de salud, fundamentalmente en las enfermeras, no siendo posible abordarlas en este trabajo por problemas de espacio.

En la literatura revisada se expone que en América Latina y el Caribe existen varios estudios en Argentina, Brasil y Costa Rica, donde éste síndrome afecta al 43-55\% de los profesionales de enfermería y al 11\% en el Perú. En la Unidad de Cuidado Crítico del Hospital Central de la Fuerza Aérea del Perú se reportó un 15\% de niveles altos en la dimensión despersonalización; y un 20\% de niveles altos en la dimensión cansancio emocional en profesionales de enfermería; sin embargo, Solìs no detecto este síndrome en el $86 \%$ de sus investigados y con tendencia a desarrollarlo en el 14\%. (Solis , Zamudio, Matzumura, \& Gutiérrez, 2016) 
Consideraciones finales:

La historia de la Seguridad Social y el Seguro Social es larga pero actualmente se considera como un logro de la sociedad moderna, ya que sus servicios no solo abarca a los trabajadores, según requisitos de cada país, sino que incluye a la población genera. Los trabajadores expuestos a riesgos que sufren accidentes o enfermedades productos de su labor son indemnizados, a ellos o a sus familiares en caso de muerte; aunque en realidad quisiéramos que no existiera necesidad de aportar esos recurso financieros a tales prestaciones sino más bien que fueran utilizados en crear las condiciones de trabajo y aplicar medidas de salud y seguridad en su puesto de trabajo.

\section{Bibliografia.}

Coromoto, P. (2015). Seguridad Social y desempeño laboral : claves de la salud y el bienestar en el Seguro Social de Venezuela Obtenido de http://mriuc.bc.uc.edu.ve/bitstream/handle/123456789/1360/cguevara.pdf?sequence=3

Huanca, R., Perretta, L., Lebensohn, N., DiTullio, L., Di Paolo, M., \& Quaglia, N. (2016). Incidencia de accidentes laborales con potencial riesgo biológico en un hospital público de tercer nivel de atención de la provincia de Santa Fe. Revsita de la Facultad de Ciencias Médicas, 73(2), 83-89.

Insitituto Nicaraguense de Seguro Social. (2018). Accidente y riegos laborales en Nicaragua. Obtenido de http://observatoriocentroamericanodeviolencialaboral.org/images/nicaragua/2Accidentes-y-riesgos-laborales-en-Nicaragua.pdf

Instituto de Seguridad Laboral. (2016). Trámites y Servicios del Instituto de Seguridad Laboral. Santiago de Chile: Ministerio de trabajo y Prevención Social.

Martin, M. (2015). Influencia de la estabilidad laboral en el desempeño del trabajador de enfermeria.Estudio realizado en el Hospital Nacional de Chimaltenango. QUETZALTENANGO: UNIVERSIDAD RAFAEL LANDÍVAR.

Moreno, J. (2016). Seguridad social obligatoria y voluntaria. México: Biblioteca Jurídica Virtual del Instituto de Investigaciones jurídicas en la UNAM.

Organización Internacional del Trabajo (OIT). (s.f.). Indemnización a los trabajadores, Vision General .Gestión y Politica. Cap 25. Enciclopedia de salud y Seguridad del Trabajo.

Ginebra.Suiza.

Obtenido de http://www.insht.es/InshtWeb/Contenidos/Documentacion/TextosOnline/EnciclopediaOIT/t omol/25.pdf 
Tatiana P. Vinces-Sornoza; Yandry M. Ponce-Menendez; Beneda M. Zambrano-Mora; Diana L. Macias-Cedeño; José C. Pinargote-Menendez; Cecilio A. Viteri-Uquillas

Padrón, Y., Moreno, S. N., Márquez, A., González , L. M., \& Pérez, F. (2017). Accidentalidad labroal en expuestos a riegos biológicos en instituciones de salud. Revista de Ciencias Médicas de Pinar de Rio, 21(2).

Porras, A. (2015). La seguridad social en Ecuadro: un necesario cambio de paradigma. Revista de derecho , 24, UASB-ECUADOR /CEN, Quito. Obtenido de http://repositorio.uasb.edu.ec/bitstream/10644/5285/1/08-TC-Porras.pdf

Rodríguez-Páez, F. G., Jiménez-Barbosa, W. G., \& Palencia-Sánchez, F. (2018). Uso de los servicios de urgencias en Bogotá, Colombia: Un análisis desde el Triaje. Universidad y Salud, 20(3), págs 215-226. Obtenido de https://dx.doi.org/10.22267/rus.182003.124)

Silva, T. D., Araújo, W., Stival, M., Toledo, A., Burke, T., \& Carregaro, R. (2018). Inconformidad musculoesqueletica, capacidad laboral y fatiga en profesionales de la enfermeria que actuan en ambiente hospitalario.Revista da Escola de Enfermagem da USP , 52 e03332 Epub. Obtenido de https://dx.doi.org/10.1590/s1980-220x2017022903332

Solis , Z., Zamudio, L., Matzumura, J., \& Gutiérrez, H. (2016). Relación entre clima organizacional y síndrome de burnout en el servicio de emergencia de un hospital Categoría III-2. Lima, Perú 2015. Horizonte Médico, 16(4), 32-38.

Vilar, M., Esteve, M., Carreras, R., Olivé, V., Bretau, F., Subirats, P., . . . Santiña , M. (2016). Estudio descriptivo de la incapacidad temporal en el sector sanitario de Cataluña (20092012). Archivo de Prevencion de Riegos Laborales, 19(1), 15-21. 